\title{
Formation of Stable Compounds of Potassium and Iron under Pressure
}

\author{
Adebayo A. Adeleke and Yansun Yao* \\ Department of Physics and Engineering Physics, University of Saskatchewan, Saskatoon, \\ Saskatchewan S7N 5E2, Canada. \\ ${ }^{*}$ Correspondence and material requests should be addressed to Y. Yao (email: \\ yansun.yao@ usask.ca)
}

Supplementary Information 
Table S1. Optimized structural information of predicted thermodynamically stable K-Fe compound that are not considered further due to dynamic instability.

Fractional atomic coordinates

\begin{tabular}{|c|c|c|c|c|c|c|c|c|c|c|c|c|}
\hline $\mathrm{P}(\mathrm{GPa})$ & SG & $a(\AA)$ & $b(\AA)$ & $c(\AA)$ & $\alpha$ & $\beta$ & $\gamma$ & Atom & $x$ & $y$ & $z$ & Site \\
\hline 30 & $P 2{ }_{1} / m$ & 10.26 & 3.77 & 2.44 & & 96.33 & & $\begin{array}{l}\mathrm{K} 1 \\
\mathrm{Fe} 1 \\
\mathrm{Fe} 3 \\
\mathrm{Fe} 5 \\
\end{array}$ & $\begin{array}{l}0.923 \\
0.470 \\
0.730 \\
0.332 \\
\end{array}$ & $\begin{array}{l}0.250 \\
0.250 \\
0.750 \\
0.750 \\
\end{array}$ & $\begin{array}{l}0.212 \\
0.735 \\
0.844 \\
0.658 \\
\end{array}$ & $\begin{array}{l}2 e \\
2 e \\
2 e \\
2 e \\
\end{array}$ \\
\hline 60 & $P 4 / n m m$ & 2.41 & 2.41 & 10.97 & & & & $\begin{array}{l}\mathrm{K} 1 \\
\mathrm{Fe} 1 \\
\mathrm{Fe} 3 \\
\end{array}$ & $\begin{array}{l}0.000 \\
0.000 \\
0.000\end{array}$ & $\begin{array}{l}0.500 \\
0.500 \\
0.500 \\
\end{array}$ & $\begin{array}{l}0.601 \\
0.207 \\
0.926 \\
\end{array}$ & $\begin{array}{l}2 c \\
2 c \\
2 c \\
\end{array}$ \\
\hline 90 & $P-1$ & 2.32 & 2.33 & 9.85 & 92.25 & 96.00 & 60.12 & $\begin{array}{c}\mathrm{K} 1 \\
\mathrm{Fe} 1 \\
\mathrm{Fe} 3 \\
\end{array}$ & $\begin{array}{l}0.000 \\
0.202 \\
0.378 \\
\end{array}$ & $\begin{array}{l}0.500 \\
0.349 \\
0.327 \\
\end{array}$ & $\begin{array}{l}0.500 \\
0.725 \\
0.092 \\
\end{array}$ & $\begin{array}{l}1 g \\
2 i \\
2 i \\
\end{array}$ \\
\hline 120 & $P-1$ & 2.29 & 2.29 & 9.63 & 92.71 & 95.73 & 95.73 & $\begin{array}{c}\mathrm{K} 1 \\
\mathrm{Fe} 1 \\
\mathrm{Fe} 3\end{array}$ & $\begin{array}{l}0.500 \\
0.873 \\
0.714 \\
\end{array}$ & $\begin{array}{l}0.500 \\
0.332 \\
0.334\end{array}$ & $\begin{array}{l}0.500 \\
0.093 \\
0.722 \\
\end{array}$ & $\begin{array}{l}1 h \\
2 i \\
2 i\end{array}$ \\
\hline 150 & $\mathrm{P}-1$ & 2.25 & 2.26 & 9.47 & 93.11 & 95.80 & 60.22 & $\begin{array}{l}\mathrm{K} 1 \\
\mathrm{Fe} 1 \\
\mathrm{Fe} 3 \\
\end{array}$ & $\begin{array}{l}0.500 \\
0.872 \\
0.718 \\
\end{array}$ & $\begin{array}{l}0.500 \\
0.335 \\
0.323 \\
\end{array}$ & $\begin{array}{l}0.000 \\
0.593 \\
0.221 \\
\end{array}$ & $\begin{array}{l}1 e \\
2 i \\
2 i\end{array}$ \\
\hline 200 & $P-1$ & 2.21 & 2.22 & 9.22 & 93.79 & 94.64 & 60.38 & $\begin{array}{l}\mathrm{K} 1 \\
\mathrm{Fe} 1 \\
\mathrm{Fe} 3\end{array}$ & $\begin{array}{l}0.000 \\
0.642 \\
0.254\end{array}$ & $\begin{array}{l}0.000 \\
0.153 \\
0.791\end{array}$ & $\begin{array}{l}0.000 \\
0.406 \\
0.218\end{array}$ & $\begin{array}{l}1 a \\
2 i \\
2 i\end{array}$ \\
\hline
\end{tabular}




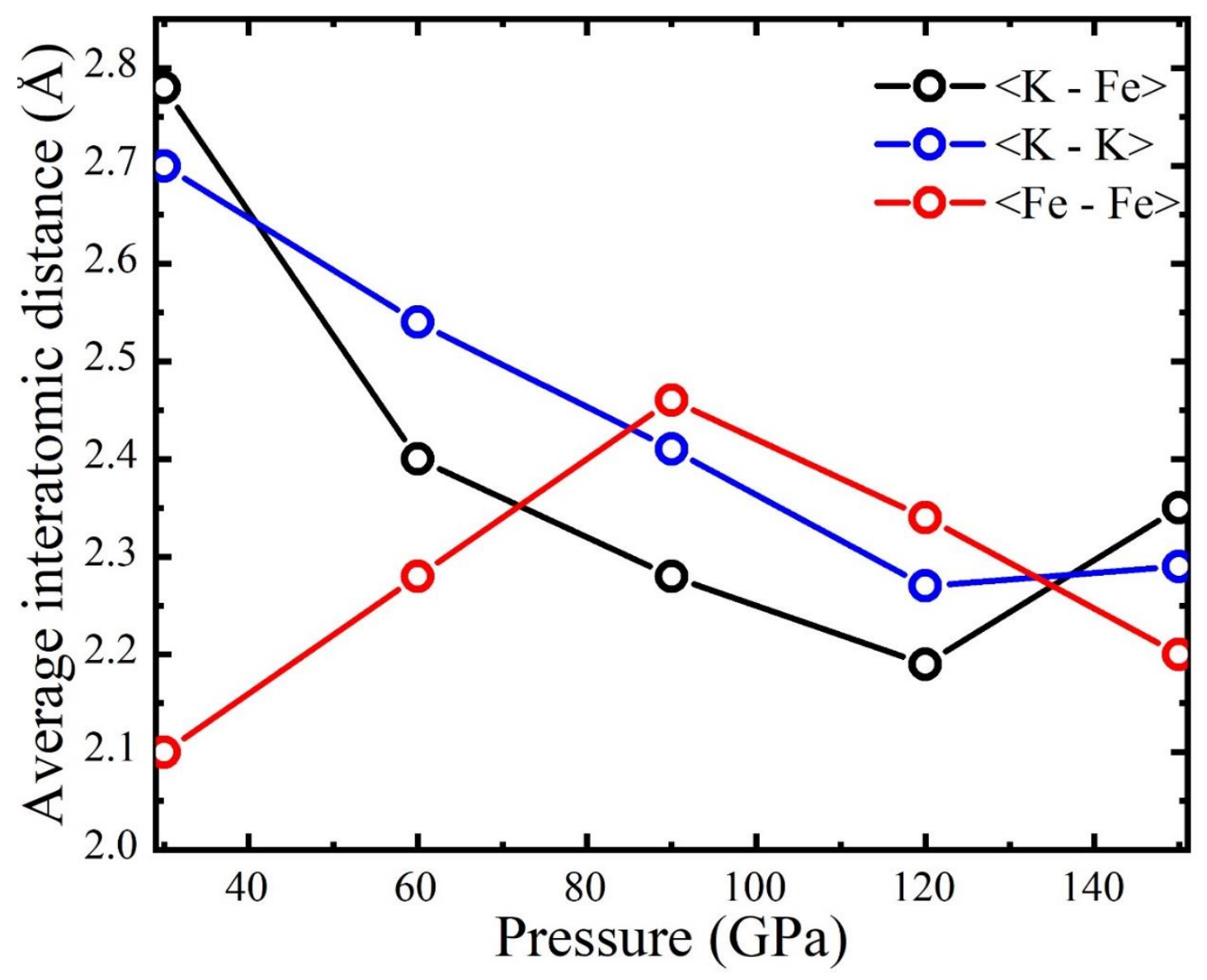

Figure S1. Interatomic distance (bondlength) evolution with pressure for various units in the KFe candidate crystal structures studied at $0 \mathrm{~K}$. 
Table S2. Bader Charge Analysis

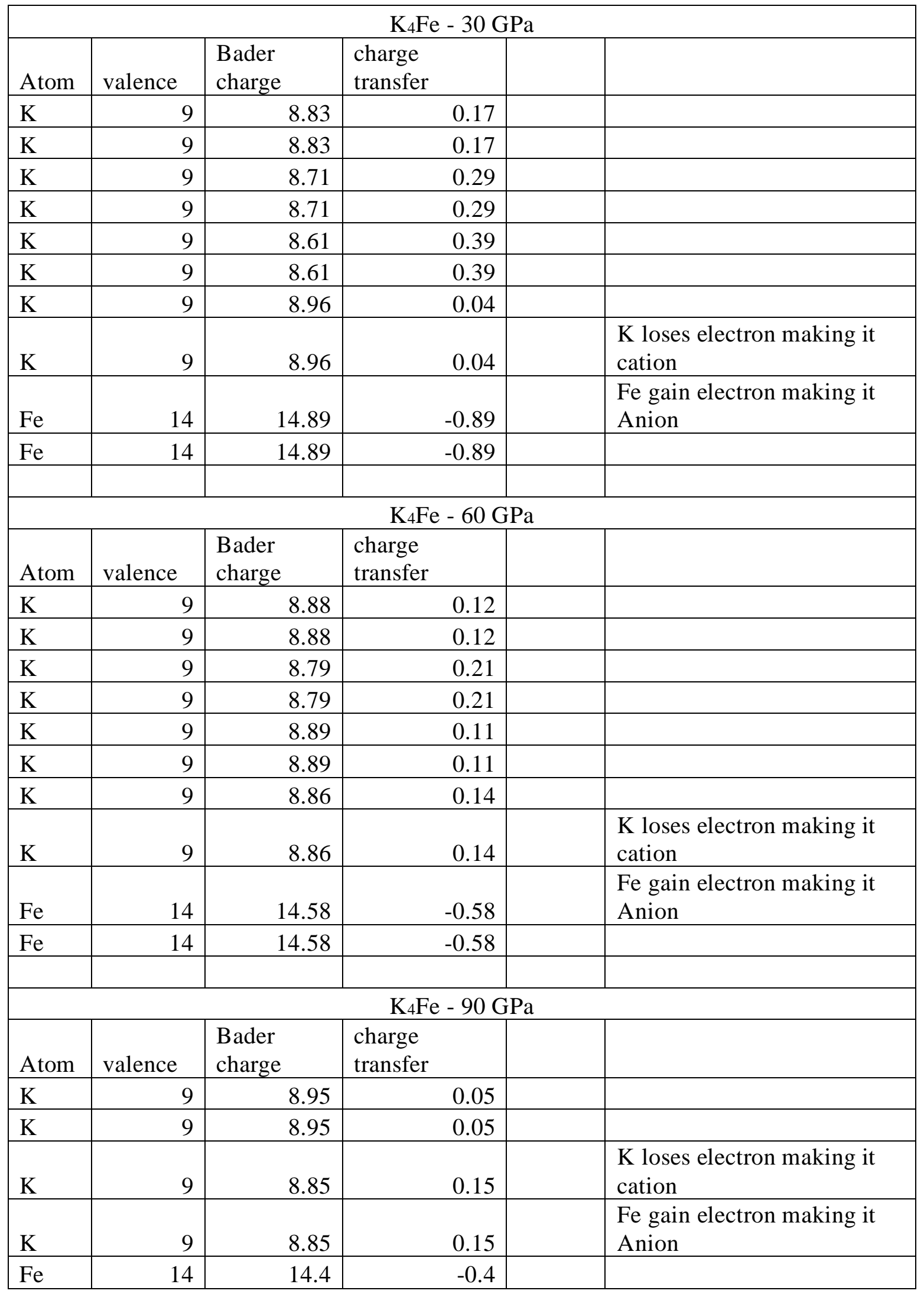




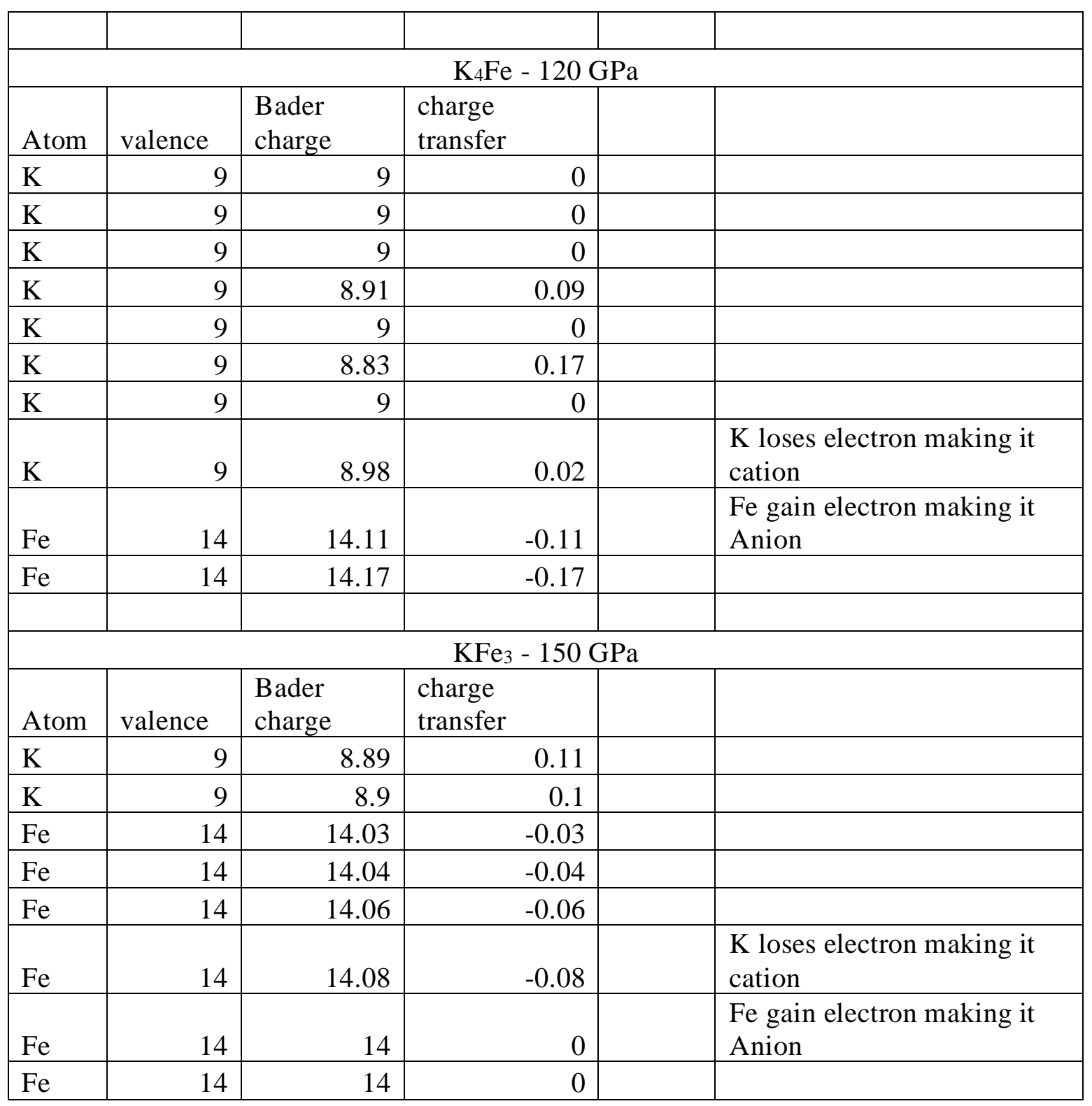



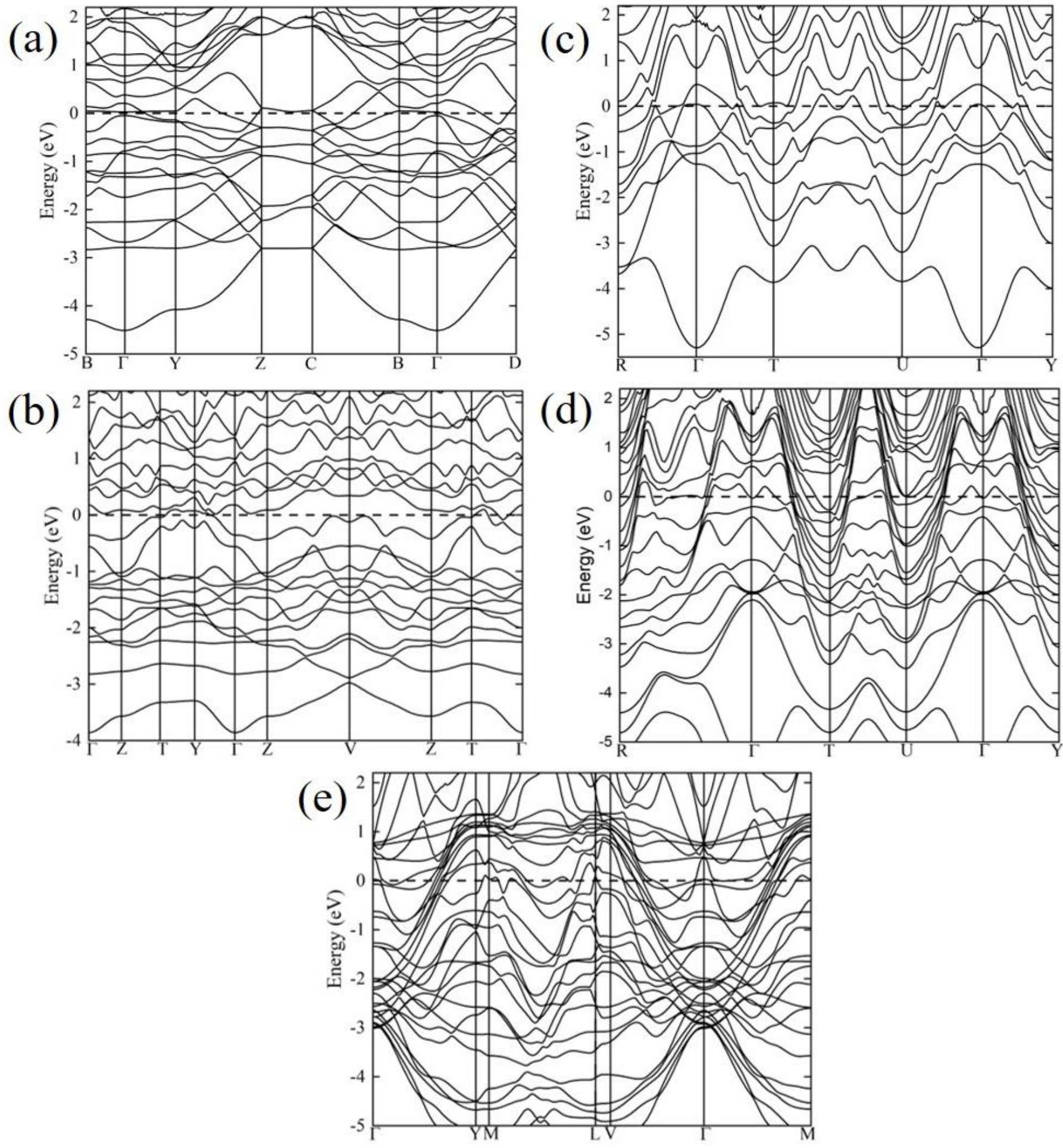

Figure S2. Calculated electronic band structure for the (a) $\mathrm{K}_{4} \mathrm{Fe}$ in $P 2{ }_{1} / m$ structure at $30 \mathrm{GPa}$ (b) $\mathrm{K}_{4} \mathrm{Fe}$ in $P-1$ structure at $60 \mathrm{GPa}$ (c) $\mathrm{K}_{4} \mathrm{Fe}\left(1\right.$ f.u.) in $P 1$ structure at $90 \mathrm{GPa}$ (d) $\mathrm{K}_{4} \mathrm{Fe}$ (2 f.u.) in $P 1$ structure at $120 \mathrm{GPa}(\mathrm{e}) \mathrm{KFe}_{3}$ in $C 2 / m$ structure at $150 \mathrm{GPa}$. The dash horizontal line is the Fermi energy level. 

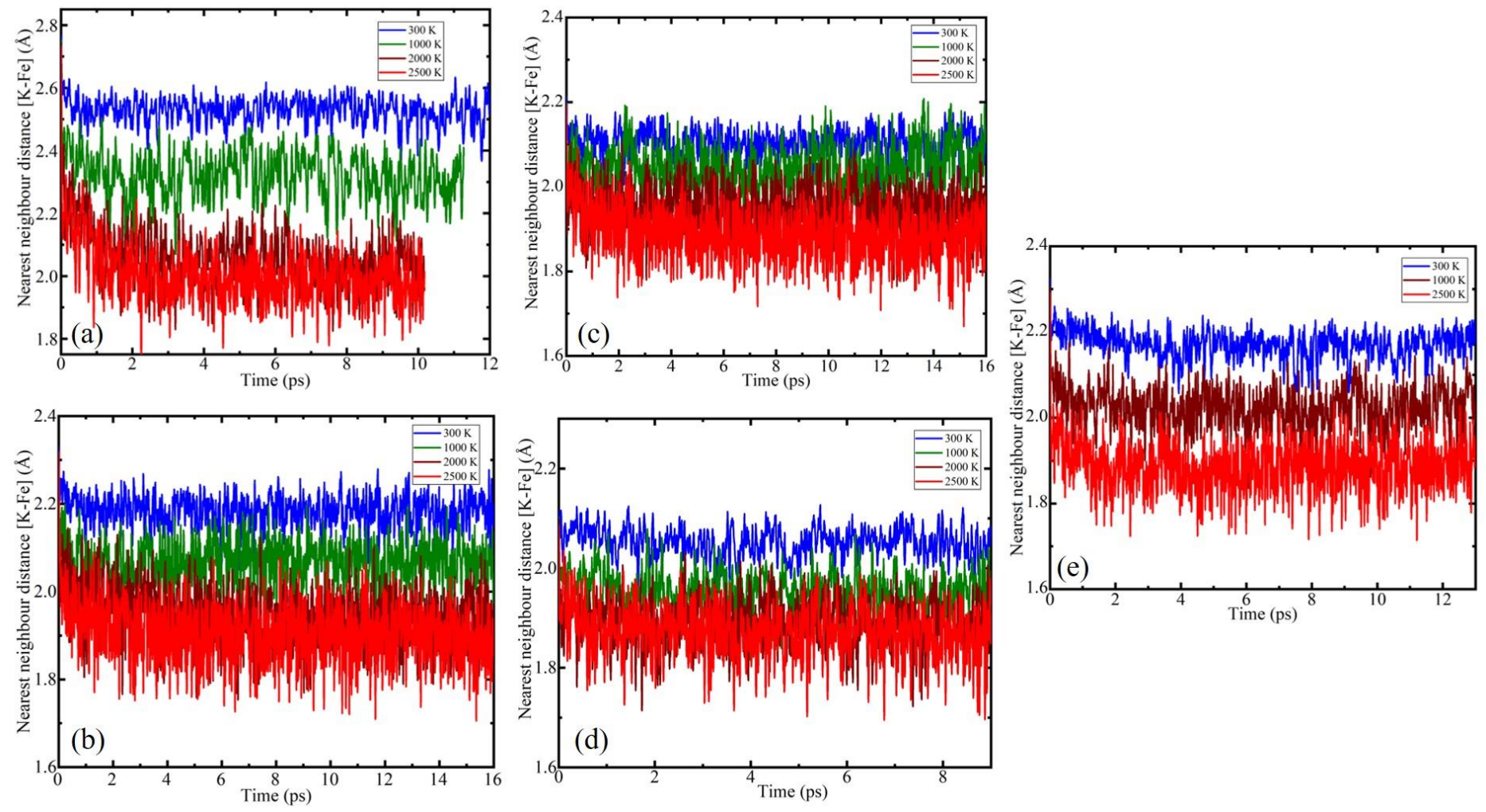

Figure S 3: Temporal evolution of the nearest neighbor distance of the (a) $\mathrm{K}_{4} \mathrm{Fe}$ in $P 2 \mathrm{l} / \mathrm{m}$ structure at $30 \mathrm{GPa}$ (b) $\mathrm{K}_{4} \mathrm{Fe}$ in $P-1$ structure at $60 \mathrm{GPa}$ (c) $\mathrm{K}_{4} \mathrm{Fe}$ ( 1 f.u.) in $P 1$ structure at $90 \mathrm{GPa}$ (d) $\mathrm{K}_{4} \mathrm{Fe}$ (2 f.u.) in $P 1$ structure at $120 \mathrm{GPa}$ (e) $\mathrm{KFe}_{3}$ in $C 2 / m$ structure at $150 \mathrm{GPa}$. The plots are shown for temperatures $300 \mathrm{~K}, 1000 \mathrm{~K}, 2000 \mathrm{~K}$ and $2500 \mathrm{~K}$. 